\title{
Urbach's rule derived from thermal fluctuations in the band-gap energy
}

\author{
Skettrup, Torben
}

Published in:

Physical Review B

Link to article, DOI:

10.1103/PhysRevB.18.2622

Publication date:

1978

Document Version

Publisher's PDF, also known as Version of record

Link back to DTU Orbit

Citation (APA):

Skettrup, T. (1978). Urbach's rule derived from thermal fluctuations in the band-gap energy. Physical Review $B$, 18(6), 2622 - 2631. https://doi.org/10.1103/PhysRevB.18.2622

\section{General rights}

Copyright and moral rights for the publications made accessible in the public portal are retained by the authors and/or other copyright owners and it is a condition of accessing publications that users recognise and abide by the legal requirements associated with these rights.

- Users may download and print one copy of any publication from the public portal for the purpose of private study or research.

- You may not further distribute the material or use it for any profit-making activity or commercial gain

- You may freely distribute the URL identifying the publication in the public portal

If you believe that this document breaches copyright please contact us providing details, and we will remove access to the work immediately and investigate your claim 


\title{
Urbach's rule derived from thermal fluctuations in the band-gap energy
}

\author{
T. Skettrup \\ Physics Laboratory III, The Technical University of Denmark, Lyngby, Denmark
}

(Received 22 February 1977)

\begin{abstract}
The exponential absorption edge (known as Urbach's rule) observed in most materials is interpreted in terms of thermal fluctuations in the band-gap energy. The main contribution to the temperature shift of the band-gap energy is due to the temperature-dependent self-energies of the electrons and holes interacting with the phonons. Since the phonon number is fluctuating in thermal equilibrium, the band-gap energy is also fluctuating resulting in an exponential absorption tail below the average band-gap energy. These simple considerations are applied to derive Urbach's rule at high temperatures, while a simplified model with independent, noninteracting atoms is proposed to explain the behavior of Urbach's rule in the whole temperature range. The three parameters entering Urbach's rule are expressed in terms of parameters derived from the temperature shift of the band gap and from the exciton absorption. Comparison with experiments is performed for the II-VI compound $\mathrm{ZnO}$. It is shown that a good agreement is found between the temperature shift of the exciton line observed experimentally and the temperature shift computed from the steepness parameter of Urbach's rule. The agreement with experimental values for the two other parameters is also satisfactory. It is shown that the band-gap shift (and absorption tail) in $\mathrm{ZnO}$ is caused by interaction with both acoustical and optical phonons. While the temperature-dependent polaron contribution can account for the optical-phonon contribution, the deformation-potential interaction with LA phonons is not sufficient to account for the acoustical-phonon contribution.
\end{abstract}

\section{INTRODUCTION}

The exponentially increasing absorption edge near the band gap observed by Urbach in $1953^{1}$ has been observed in many semiconductors and seems to be a fundamental optical property of the solid state. A review has been given by Kurik, ${ }^{2}$ and many theories have been proposed to explain Urbach's rule. ${ }^{2-4}$ By now, the most accepted theories are probably Dow and Redfield's microfield theory ${ }^{3}$ or Sumi and Toyozawa's theory of the momentarily trapped exciton. ${ }^{4}$

It is the purpose of the present work to give a general derivation of Urbach's rule independent of the nature of the electron-lattice interaction causing this low-energy absorption tail. This can be done by ascribing the absorption tail to the thermal fluctuations in the band-gap energy. In Sec. II simple arguments are presented leading to the well-known expression for Urbach's rule at high temperatures, but expressed in terms of the band-gap temperature shift. In Sec. III a specific model is proposed from which the exponential absorption edge is obtained in the whole temperature range. In Sec. IV the theory is compared with experimental results on band-gap shift and absorption edge of $\mathrm{ZnO}$.

\section{BASIC CONSIDERATIONS}

In thermal equilibrium at temperature $T$ the ave rage number of phonons in the $j$ th mode is given by the Bose-Einstein factor

$$
\left\langle n_{j}\right\rangle=\left[\exp \left(\hbar \omega_{j} / k T\right)-1\right]^{-1},
$$

where $\hbar \omega_{j}$ is the phonon energy and $k T$ the thermal energy. It is well known that Bose-Einstein fields exhibit very large fluctuations. At temperature $T$, the standard deviation of the occupation number $n_{j}$ is given by :

$$
\sigma_{n j}=\left(\left\langle n_{j}^{2}\right\rangle-\left\langle n_{j}\right\rangle^{2}\right)^{1 / 2}=\left\langle n_{j}\right\rangle\left[1+\left(1 /\left\langle n_{j}\right\rangle\right)\right] .
$$

Hence, $\sigma_{n j}$ is never less than the average occupancy $\left\langle n_{j}\right\rangle$ and at low temperatures $\sigma_{n j}$ will even be considerably larger than $\left\langle n_{j}\right\rangle$.

In a simple model of a crystal the phonon field is considered coherent through out the whole crystal volume. In a more realistic model, however, the coherence lengths of the phonons must be taken into account. As estimated from phonon lifetimes deduced from reststrahlen spectra ${ }^{6}$ the phonon coherence lengths are of the order of 10_ 100 wavelengths. Hence, the crystal should be divided up into small cells of this size for each phonon mode. On the average, over all the cells, the occupancy is given by (1), but the local fluctuations in the individual cells may be very large as indicated in (2). The effect of the phonon field on the absorption of light in a solid is governed by the electron-phonon interaction, which implies change of electron energy (self-energy correction), scattering of electrons (broadening of absorption lines), and perturbation of electron wave functions (change in absorption strength).

In the present section we shall concentrate on the self-energy correction and neglect the other two contributions. The temperature-dependent self-energy corrections for the electron and hole energies give rise to the main contribution to the 
band-gap shift with temperature (typical about $80 \%){ }^{7,8}$ The rest of the band-gap shift is due to the crystal expansion. The self-energy correction is proportional to the average number of phonons $\left\langle n_{j}\right\rangle$ given by (1). ${ }^{7}$ For the sake of simplicity we assume that the band-gap shift due to the crystal expansion is proportional to $\left\langle n_{j}\right\rangle$, too.

This simplification is probably not so severe since the crystal expansion only contribute about $20 \%$ to the total band-gap shift with temperature. In this case, the energy gap $E_{g}(T)$ as a function of temperature $T$ is given by

$$
E_{g}(T)=E_{0}-\sum_{j} A_{j}\left\langle n_{j}\right\rangle,
$$

where $E_{0}$ is the zero-temperature band gap, and the proportionality constants $A_{j}$ include contributions from both the electron and hole self-energies in the vibrating lattice and the crystal expansion. In order to show in a simple manner how the fluctuations in the phonon concentration can give rise to an exponential absorption edge, we shall make the simplifying assumption that the vibrating lattice can be represented by a single set of oscillators with one phonon energy $\hbar \omega$. In this case (3) can be written for a three-dimensional lattice

$E_{g}(T)=E_{0}-A\left(\left\langle n_{x}\right\rangle+\left\langle n_{y}\right\rangle+\left\langle n_{z}\right\rangle\right)=E_{0}-3 A\langle n\rangle$.

The averaging in (4) must be carried out over times much longer than the phonon lifetimes and space regions much larger than the phonon coherence lengths. Inside a cell of a size given by the phonon coherence length during a time given by the phonon coherence time (phonon lifetime), we can find a local and instantaneous value $E_{g, 10 c}$ of the band gap

$$
E_{g, 10 \mathrm{c}}=E_{0}-A\left(n_{x}+n_{y}+n_{z}\right),
$$

where $\left(n_{x}, n_{y}, n_{z}\right)$ are the occupancy number of phonons in that particular cell during that particular time. This means that there are thermal fluctuations in the band-gap energy throughout the crystal as well as there are thermal fluctuations in the phonon concentration. These thermal fluctuations are relevant for photon absorption since they occur much slower than the photon propagation through the individual cells of the crystal. The time it takes for a photon to propagate through such a cell is much smaller than the phonon lifetime because the speed of light is much greater than the phase velocity of the phonons. In the case of photon propagation, then, the phonon population in the individual cells will seem frozen, and the crystal will act as an inhomogeneous medium composed of regions with different band gaps. The average band gap of the crystal is given by (4), and the standard deviation is found by combining (2) and (4).

These fluctuations in the energy gap immediately explain the presence of an exponentially increasing absorption edge as seen in the following, and they also explain the generality of Urbach's rule, since such thermal fluctuations are present in all solids due to the Bose-Einstein statistics of the phonon field.

The probability that a particular cell contains the particular number $\left(n_{x}, n_{y}, n_{z}\right)$ of phonons in (5) is given by the distribution function for bosons ${ }^{5}$ $p_{n}=\exp \left[-\left(n_{x}+n_{y}+n_{z}\right) \hbar \omega / k T\right][1-\exp (-\hbar \omega / k T)]^{3}$.

From (5) and (6) we find

$p_{n}=\exp \left[-\hbar \omega\left(E_{0}-E_{g_{0} 10 c}\right) / A k T\right][1-\exp (-\hbar \omega / k T)]^{3}$.

The absorption coefficient $\alpha(h \nu)$ at the photon energy $h \nu$ must be proportional to $p_{n}$ with $E_{g, 10 c}$ replaced by $h \nu$ (i.e., the probability that a photon with energy $h \nu$ finds a local energy gap $E_{g, 10 c}$ $=h \nu$ and therefore is absorbed)

$$
\alpha(h \nu)=\alpha_{0} \exp \left[-\sigma\left(E_{0}-h \nu\right) / k T\right],
$$

where $\alpha_{0}$ is a weakly temperature-dependent proportionality constant and

$$
\sigma=\hbar \omega / A \text {. }
$$

The constant $A$ is obtained from the shift of the band gap at higher temperatures

$$
E_{g}(T) \simeq E_{0}-A 3 k T / \hbar \omega,
$$

where the high-temperature limit of (1) has been introduced into (4) to obtain a linear band-gap shift with temperature. Taking the derivative of (10)

$$
\frac{d E_{g}}{d T}=-\frac{3 k A}{\hbar \omega},
$$

we finally obtain the connection between the steepness parameter $\sigma$ and the band-gap shift

$$
\sigma=3 k /\left(-\frac{d E_{g}}{d T}\right) .
$$

Equations (8) and (11) describe an exponentialiy increasing absorption edge following the empirical rule of Urbach, ${ }^{1}$ but where the logarithmic slope $\sigma$ is expressed in terms of the temperature shift of the band gap without reference to any specific electron-phonon interaction.

A useful expression for the absorption coefficient is obtained by combining (8)-(10):

$$
\alpha=\alpha_{0} \exp \left\{-3\left(E_{0}-h \nu\right) /\left[E_{0}-E_{g}(T)\right]\right\},
$$

which although derived for the high-temperature case appears to be valid also for lower tempera- 
tures [see Eq. (37)].

The well-known temperature dependence of the steepness parameter

$$
\sigma=\sigma_{0}(2 k T / \hbar \omega) \tanh (\hbar \omega / 2 k T)
$$

cannot be derived from the simple arguments given above, but is obtained in Sec. III for a more specific model.

\section{MODEL FOR ABSORPTION EDGE}

As indicated in Sec. II an exponential absorption edge can arise from the local fluctuations in the energy gap due to the thermal phonon field. The simple considerations applied there failed, however, to predict the correct low-temperature dependence of the absorption edge. In the present section we shall introduce a simple model from which Urbach's rule can be derived in the whole temperature range.

In Sec. II it was shown that the crystal should be divided up into microscopic cells of a size of the phonon coherence length. In the present model we shall reduce this cell size to the size of the unit cell. Hence, the interaction between the atoms is neglected, and the crystal is considered to consist of completely independent and noninteracting identical atoms. The phonons then correspond to the excited vibrational quanta of the individual atoms. The atom in the unit cell is considered to have two energy levels which are separated by an energy distance equal to the temperature-dependent band-gap energy of the real crystal. As wave functions for the two levels one can use the Wannier functions for the real crystal

$$
\phi_{c, v}\left(\overrightarrow{\mathrm{r}}-\overrightarrow{\mathrm{a}}_{p}\right)=\frac{1}{(N)^{1 / 2}} \sum_{\overrightarrow{\mathrm{k}}} e^{-i \overrightarrow{\mathrm{k}} \cdot \overrightarrow{\mathrm{a}}_{p}} \psi_{\overrightarrow{\mathrm{k}}, c, v}(\overrightarrow{\mathrm{r}}),
$$

where $\vec{a}_{p}$ is the position of the pth unit cell, $N$ is the number of unit cells, and $\psi_{\overrightarrow{\mathrm{k}}, c, v}(\overrightarrow{\mathrm{r}})$ are the usual Bloch functions with wave vector $\vec{k}$ for the conduction band $(c)$ or valence band $(v)$. These Wannier functions are localized within the $p$ th unit cell.

In the harmonic approximation the vibration of the $p$ th atom is described by the harmonic-oscillator wave function

$$
\chi_{n i}\left(\Delta_{p i}\right)=N_{n i} H_{n i}\left(\Delta_{p i} / \alpha\right) \exp \left(-\Delta_{p i}^{2} / 2 \alpha\right),
$$

where $\Delta_{p i}$ is the $i$ th component of the $p$ th atomic displacement $\vec{\Delta}_{p}=\vec{a}-\vec{a}_{p} \cdot N_{n i}$ is the usual normalizing constant, $H_{n i}$ is the Hermite polynomium of $n_{i}$ th degree, and $\alpha^{2}=\hbar / M \omega$, where $M$ is the oscillating mass and $\omega$ its angular frequency. For the sake of simplicity only one frequency is considered.

Without interaction between electron and lattice the total wave function for the electron-vibrating atom system in the $p$ th unit cell is just the product

$$
\Phi_{c, v}^{p}\left(\overrightarrow{\mathrm{r}}, \vec{\Delta}_{p}\right)=\phi_{c, v}\left(\overrightarrow{\mathrm{r}}-\overrightarrow{\mathrm{a}}_{p}\right) \chi_{n x}\left(\Delta_{p x}\right) \chi_{n y}\left(\Delta_{p y}\right) \chi_{n z}\left(\Delta_{p z}\right),
$$

and the total energy is just the sum of the electron energy and the atomic vibrational energy $\left(n_{x}+n_{y}+n_{z}+\frac{3}{2}\right) \hbar \omega$. The absorption coefficient for the atom is given by

$$
\begin{aligned}
\alpha & =C_{0}\left|\left\langle\phi_{v}\left|H_{r}\right| \phi_{c}\right\rangle\right|^{2} \delta\left(h \nu-E_{g 0}\right) \\
& =C_{0}\left|\mu_{v c}\right|^{2} \delta\left(h \nu-E_{g 0}\right)
\end{aligned}
$$

where $C_{0}$ is a proportionality constant and $\mu_{v c}$ is the dipole matrix element for the transition between the two energy levels of the atom. The absorption line has a $\delta$-function shape around $E_{g 0}$ which is the zero-temperature band-gap energy (or rather the zero-temperature exciton groundstate energy of the real crystal).

When the electron-lattice interaction is switched on both (16) and (17) change. We shall not be concerned here with what kind of interaction is present, but only consider the effects of the interaction, which are perturbations of energy levels and wave functions. This leads to a different bandgap energy and a different dipole matrix element $\mu_{v_{c}}$ in (17). As discussed in Sec. II this different band-gap energy should be introduced as a fluctuating local energy gap with the instantaneous value

$E_{g, 10 c}=E_{\mathrm{g} 0}-A_{c}\left(n_{c x}+n_{c y}+n_{c z}\right)-A_{v}\left(n_{v x}+n_{v y}+n_{v z}\right)$.

Here, the self-energies have been divided up into two parts since both the electron and the hole of the real crystal gain energy from the interaction with the lattice. Similarly, the atomic vibrational energy is changed into $\left(n_{c x}+n_{c y}+n_{c z}+\frac{3}{2}\right) \hbar \omega_{c}$ when the electron is in the upper level and $\left(n_{v x}+n_{v y}+n_{v z}\right.$ $\left.+\frac{3}{2}\right) \hbar \omega_{v}$ when it is in the lower level. The difference between $\omega_{c}$ and $\omega_{v}$ arises from different contributions to the phonon self-energy from the interactions with the electrons and holes. In the following, however, we shall neglect this difference and put $\hbar \omega_{c}=\hbar \omega_{v}=\hbar \omega$.

The absorption coefficient for the $p$ th atom is now given by

$$
\begin{aligned}
\alpha_{1 \mathrm{oc}}=C_{0} \sum_{\substack{n_{c x,} n_{c y}, n_{c z} \\
n_{v x}, n_{v y}, n_{v z}}} p\left(n_{v x}, n_{v y}, n_{v z}\right) \\
\times\left|\left\langle\Phi_{v}\left|\hat{H}_{r}\right| \Phi_{c}\right\rangle\right|^{2} \delta\left(h \nu-E_{g, 1 \mathrm{oc}}\right),
\end{aligned}
$$

where $p\left(n_{v x}, n_{v y}, n_{v z}\right)$ is the distribution function (6) for the excited vibrational quanta in thermal equilibrium; $\Phi_{c}$ and $\Phi_{v}$ are the total wave functions for the interacting electron-vibrating atom system. 
Since $\Phi_{c}$ and $\Phi_{v}$ are unknown we shall use a firstorder approximation where the total wave function is still written as a product of electron and oscillator functions as in (16), but where the electron functions are replaced by the perturbed electron wave functions. In this case the absorption coefficient (19) can be written

$$
\begin{aligned}
\alpha_{10 c}=C_{0}\left|\mu_{v c}^{\prime}\right|^{2} \sum_{n_{v}, n_{c}} p\left(n_{v}\right) \\
\quad \times\left|\left\langle\chi_{n v}(\vec{\Delta}) \mid \chi_{n c}(\vec{\Delta})\right\rangle\right|^{2} \delta\left(h \nu-E_{g, 10 c}\right)
\end{aligned}
$$

where $\mu_{v c}^{\prime}$ is the new dipole matrix element computed with the perturbed electron wave function; $n_{c}$ and $n_{v}$ are short-hand notations for the three vibrational quantum numbers. Without electronlattice interaction the overlap integral in (20) is just the Kronecker delta $\delta_{n c, n v}$. With electronlattice interaction the wave functions are changed. Instead of computing this change from perturbation theory, it is simpler to apply the approximation of displaced oscillators. In this approximation the oscillator functions are kept unchanged, but the average position of the vibrating atom is displaced a small amount $\vec{\Delta}_{0}$ when an electron transition from the lower to the upper energy level occurs. This displacement is due to the slightly different interaction forces between neighboring atoms for the two different electronic configurations. This approximation is commonly applied in connection with absorption or emission processes in deep centers (configurational coordinate mo$\mathrm{del}^{9}$ ) and in polaron theory. ${ }^{10}$

The calculation of the overlap integral in (20) can be simplified considerably in the present case because $n_{v}$ and $n_{c}$ must be relatively large numbers since the exponential absorption tail extends far below the zero-temperature band gap (up to 0.5 $\mathrm{eV}$ at higher temperatures). Hence, the oscillator behaves nearly classically, and the probability density for the position of the oscillating atom is strongly peaked near the classical reversal points for the vibration. In particular, the quantum numbers $n_{c x}, n_{c y}$, and $n_{c z}$ of the final state can be large, since the final state can be far from the thermal equilibrium states. We shall the refore approximate the final-state oscillator functions $\chi_{n c}\left(\vec{\Delta}-\vec{\Delta}_{0}\right)$ by $\delta$ functions peaked at the classical reversal points $\vec{\Delta}_{n c}$ determined from

$$
\frac{1}{2} M \omega^{2}\left(\vec{\Delta}_{n c}-\vec{\Delta}_{0}\right)^{2}=\left(n_{c x}+n_{c y}+n_{c z}+\frac{3}{2}\right) \hbar \omega,
$$

which is valid for large quantum numbers, and where $\vec{\Delta}_{0}$ is the small displacement of the average atomic position previously described. (As shown in Appendix B $\Delta_{0}$ is of the order of $\frac{1}{20} \AA$ for ZnO.) In this approximation, we obtain

$$
\begin{array}{rl}
\alpha_{1 \mathrm{oc}}=C_{0}\left|\mu_{v c}^{\prime}\right|^{2} \sum_{n_{v, n_{c}}} & p\left(n_{v}\right) \\
& \times\left|\chi_{n v}\left(\vec{\Delta}_{n c}\right)\right|^{2} \delta\left(h \nu-E_{g, 10 c}\right) .
\end{array}
$$

The sum over $n_{c}$ can be converted into an integral

$$
\alpha_{1 \mathrm{oc}}=C_{0}\left|\mu_{v c}^{\prime}\right|^{2} 8 \sinh ^{3} \frac{\hbar \omega}{2 k T} \int_{\vec{\Delta}_{n c}} \prod_{i=1}^{3} \sum_{n_{i}=0}^{\infty} \exp \left(-\left(n_{i}+\frac{1}{2}\right) \frac{\hbar \omega}{k T}\right) \chi_{n i}^{2}\left(\Delta_{n_{c i}}-\Delta_{0}\right) \delta\left(h \nu-E_{g, 1 \mathrm{oc}}\right) d^{3} \vec{\Delta}_{n c},
$$

where (6) has been used. The sum over $n_{i}$ is given by Mehler's formula ${ }^{9}$

$$
\sum_{n=0}^{\infty} \exp \left(-\left(n+\frac{1}{2}\right) \frac{\hbar \omega}{k T}\right)\left|\chi_{n}(\Delta)\right|^{2}=\left(2 \pi \alpha^{2} \sinh \frac{\hbar \omega}{k T}\right)^{-1 / 2} \exp \left(-\frac{\Delta^{2}}{\alpha^{2}} \tanh \frac{\hbar \omega}{2 k T}\right) \text {. }
$$

In order to perform the integration in (23) $E_{g, 10 c}$ in (18) must be expressed in terms of $\vec{\Delta}_{n c}$. In (21) the relationship between $n_{c}$ and $\vec{\Delta}_{n c}$ has already been found. Similarly, a relationship between $n_{v}$ and $\vec{\Delta}_{n c}$ can be written

$$
\frac{1}{2} M \omega^{2} \vec{\Delta}_{n c}^{2}=\left(n_{v_{x}}+n_{v_{y}}+n_{v_{z}}+\frac{3}{2}\right) \hbar \omega
$$

which is valid for large quantum numbers, and where it has been used that the instantaneous electronic transition energy $E_{g, 10 c}$ in (18) should be computed at the instantaneous reversal point $\vec{\Delta}_{n c}$ of the atom due to the $\delta$-function approximation of the oscillator function described above. (In order to prove this the time-dependent wave functions must be introduced in the calculation of the transi- tion probability, and it must be used that the time variations of the oscillator functions is much slower than for the electronic wave functions.)

The approximations used above are essentially analogous to the old Franck-Condon principle (see, for example, Ref. 9) stating that an electronic transition in a vibrating atom occurs so fast that the position and momentum of the vibrating atom do not change appreciably during the transition. Since the vibrating atom stays the longest time at the reversal points most electronic transitions occur there.

Combining (18), (21), and (25) we obtain

$$
E_{g, 10 c}=E_{0}+F \vec{\Delta}_{0} \cdot \vec{\Delta}_{n c}-G \vec{\Delta}_{n c}^{2},
$$

where 


$$
\begin{aligned}
& E_{0}=E_{g 0}+\frac{3}{2}\left(A_{c}+A_{v}\right)-A_{c}(M \omega / 2 \hbar) \vec{\Delta}_{0}^{2}, \\
& F=A_{c}(M \omega / \hbar), \\
& G=(M \omega / 2 \hbar)\left(A_{c}+A_{v}\right) .
\end{aligned}
$$

In order to obtain the exponentially increasing absorption edge the quadratic term in (26) must be larger than the linear term in the relevant spectral range. This happens for vibrational amplitudes

$$
\left|\Delta_{n c}\right| \gg F \Delta_{0} / G=2 A_{c} \Delta_{0} /\left(A_{c}+A_{v}\right)
$$

or, from (26), for

$$
\begin{aligned}
E_{g, 10 c} \ll & E_{0}-4 A_{c}\left(M \omega \Delta_{0}^{2} / \hbar\right) A_{c} /\left(A_{c}+A_{v}\right) \\
= & E_{g 0}+\frac{3}{2}\left(A_{c}+A_{v}\right) \\
& -\frac{1}{2} A_{c}\left(M \omega \Delta_{0}^{2} / \hbar\right)\left(A_{v}+9 A_{c}\right) /\left(A_{c}+A_{v}\right) .
\end{aligned}
$$

It is shown in Appendix $\mathrm{B}$ (for the case of $\mathrm{ZnO}$ ) that (31) is fulfilled for local energy gaps more than $25 \mathrm{meV}$ below $E_{g 0}$. For the spectral range of the absorption tail which at higher temperatures extends more than $0.5 \mathrm{eV}$ below $E_{g 0}$ the linear term in (26) can thus be neglected.

Performing the integration in (23) we then find using (24) and (26) that

$$
\alpha_{1 \mathrm{oc}}=\alpha_{0} \exp \left(-\left(E_{0}-h \nu\right) \tanh \frac{\hbar \omega}{2 k T} / G \alpha^{2}\right),
$$

where $\alpha_{0}$ is slightly temperature dependent. Equation (32) has been derived for the pth unit cell. In the present model, however, the atoms in the different unit cells are independent of each other. Hence, each of the unit cells gives similar contributions, and the absorption coefficient for the whole crystal is therefore of the same form as in (32), describing an exponentially increasing absorption edge below the band gap. This absorption tail arises from the thermal fluctuations in the bandgap energy. The three parameters $\alpha_{0}, E_{0}$, and $\sigma$ of Urbach's rule (8) can now be given an interpretation. $E_{0}$ is given by (27). It is seen that $E_{0}$ is somewhat shifted from $E_{g 0}$ as also observed experimentally. It is shown in Appendix B that $\alpha_{0}$ is the maximum absorption coefficient at the center of the absorption line representing the band gap (exciton line of the real crystal). As seen from (32) the $\sigma$ parameter is now given by

$$
\sigma=\frac{k T}{G \alpha^{2}} \tanh \frac{\hbar \omega}{2 k T}=\sigma_{0} \frac{2 k T}{\hbar \omega} \tanh \frac{\hbar \omega}{2 k T},
$$

with

$$
\sigma_{0}=\frac{\hbar \omega}{2 G \alpha^{2}}=\frac{\hbar \omega}{A_{c}+A_{c}}=3 k /\left(-\frac{d E_{g}}{d T}\right),
$$

where $-d E_{g} / d T$ is the high-temperature slope of the band-gap shift with temperature as obtained from the thermal average of (18)

$$
\begin{aligned}
E_{g}(T) & =E_{g 0}-3\left(A_{c}+A_{v}\right)\langle n\rangle \\
& =E_{0}^{\prime}-\frac{3}{2}\left(A_{c}+A_{v}\right) \operatorname{coth} \hbar \omega / 2 k T,
\end{aligned}
$$

with

$$
E_{0}^{\prime}=E_{g 0}+\frac{3}{2}\left(A_{c}+A_{v}\right) .
$$

By means of (34) and (35) it is now possible to rewrite (32) in the form

$$
\alpha=\alpha_{0} \exp \left\{-3\left(E_{0}-h \nu\right) /\left[E_{0}^{\prime}-E_{g}(T)\right]\right\},
$$

which is equivalent to Eq. (12) derived in Sec. II It should be noted that the temperature dependence of $\sigma$ in (33) (which could not be obtained in Sec. II) is the result of the explicit introduction of the harmonic-oscillator functions in the summation over thermally populated states in Eq. (24).

\section{COMPARISON WITH EXPERIMENTS}

The comparison with experimental results will be carried out for the hexagonal II-VI compound ZnO for which there exist data for both band-gap shift ${ }^{11}$ and absorption edge. ${ }^{12}$ The experimentally observed band-gap shift with temperature for the $C$ exciton in $\mathrm{ZnO}$ is shown in Fig. 1. In Secs. I-III the contribution from the crystal expansion to the band-gap shift was treated as a self-energy correction. This crude approximation makes it worthwhile to separate the two contributions when the

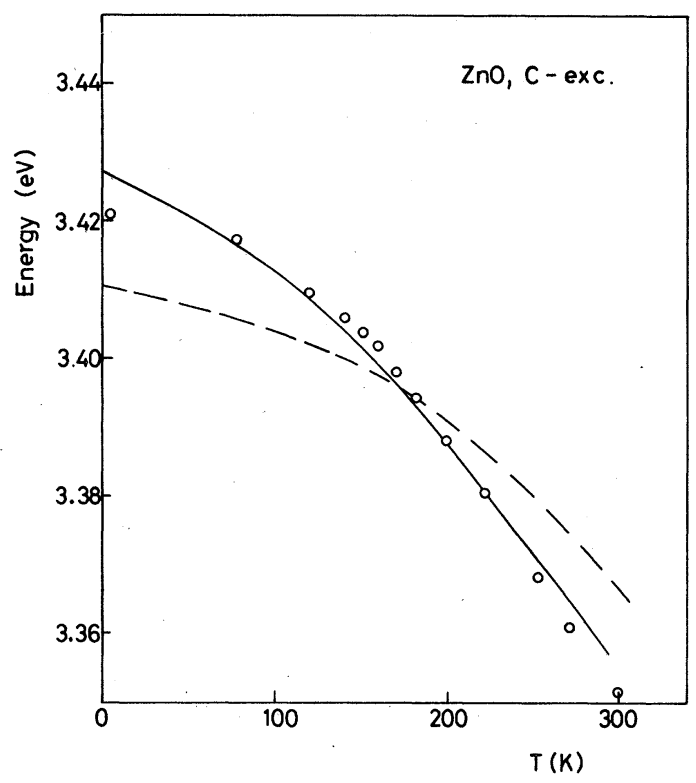

FIG. 1. Temperature dependence of the $C$-exciton line in $\mathrm{ZnO}$. The experimental points are taken from Ref. 11. The dashed curve is computed from Eq. (40) by means of the experimental values for $\sigma(T)$ shown in Fig. 3. The solid curve is computed from Eq. (43) by means of the two-phonon parameter set given in Ref. 12. 


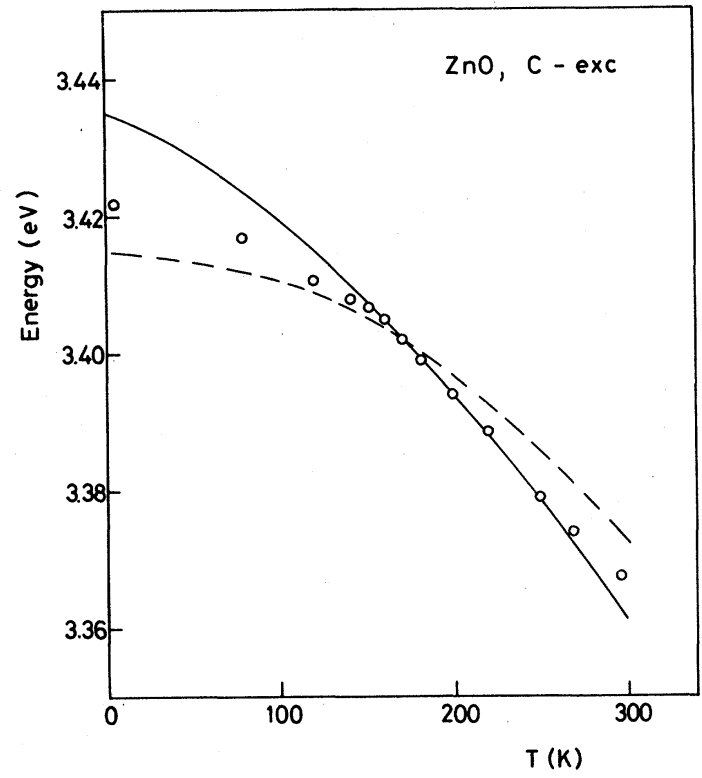

FIG. 2. Temperature shift of the $C$ exciton in $\mathrm{ZnO}$ due to the temperature-dependent self-energy corrections for the electrons and holes. The points are obtained from the experimental points in Fig. 1 by subtracting the contribution to the band-gap shift from the crystal expansion. The dashed curve is computed from Eq. (40) by means of the experimental values of $\sigma(T)$ in Fig. 3. The solid curve is computed from Eq. (43) by means of the two-phonon parameter set given in Ref. 12 .

comparison with the experimental results is carried out. The contribution $\Delta E_{g}(T)$ from the crystal expansion can be estimated by means of the temperature-dependent expansion coefficients $\alpha_{1}(T)$ and $\alpha_{11}(T)$ (Ref. 13) (perpendicular and parallel to the $c$ axis) in the following way:

$$
\Delta E_{g}(T)=C \Delta V / V=C\left[2 \alpha_{\perp}(T)+\alpha_{11}(T)\right] T,
$$

where $\Delta V / V$ is the fractional volume expansion, and $C$ is an average deformation potential constant which is obtained from the hydrostatic pressure measurements of Knell and Langer ${ }^{14}$

$$
C=(1 / S) \Delta E_{g} / \Delta p=4.0 \mathrm{eV} .
$$

Here,

$$
S=2\left(S_{11}+S_{12}+2 S_{13}\right)+S_{33}=6.836 \times 10^{-7} \text { bar }^{-1}
$$

is an appropriate sum of compliance constants, ${ }^{15}$ and $\Delta E_{g} / \Delta p=2.7 \times 10^{-6} \mathrm{eV} / \mathrm{bar}$ for the $C$ exciton of $\mathrm{ZnO} .^{14}$ The contribution (38) from the crystal expansion with the temperature-dependent expansion coefficients from Ref. 13 introduced can be subtracted from the total band-gap shift in Fig. 1. This is done in Fig. 2, where the experimental points then describe the band-gap shift arising from the temperature dependent part of the selfenergies of the electrons and holes. As shown in
Appendix A neither the self-energies from the deformation potential coupling nor from the polaron coupling are sufficient to give the correct magnitude of the band-gap shift observed in $\mathrm{ZnO}$.

An exponentially increasing absorption edge has been observed in $\mathrm{ZnO}^{12}$ and the parameters of Urbach's rule (8) were derived from these experimental results, $\alpha_{0}=4.5 \times 10^{6} \mathrm{~cm}^{-1}, E_{0}=3.51 \mathrm{eV}$, $\sigma_{0}=0.90, \hbar \omega=30 \mathrm{meV}$, or under the assumption that two different phonon modes contribute, ${ }^{12}$ $\sigma_{01}=0.90, \hbar \omega_{1}=17 \mathrm{meV}$ and $\sigma_{02}=2.20, \hbar \omega_{2}=72$ $\mathrm{meV}$. The temperature dependence observed for the $\sigma$ parameter is shown in Fig. 3. Comparison between (8), (36), and (37) shows that the bandgap shift can be expressed in terms of the temperature-dependent steepness parameter $\sigma(T)$ in the following way:

$$
E_{g}(T)=E_{g 0}+\frac{3}{2}\left(A_{c}+A_{v}\right)-3 k T / \sigma(T),
$$

where $E_{g 0}$ is the band gap at zero temperature. A value of $\left(A_{c}+A_{v}\right)=33.3 \mathrm{meV}$ is obtained from (34), when the one-phonon parameter set given above is used.

From the experimental values of $\sigma(T)$ given in Fig. 3 the band-gap shift $E_{g}(T)$ expected according to (40) can be obtained. This is shown in Figs. 1 and 2 as the dashed curves. Both curves were shifted somewhat along the energy axis to obtain best fit. This corresponds to using $\frac{3}{2}\left(A_{c}+A_{v}\right)$ as a fitting parameter. It turns out that the best fit is obtained using $0.92\left(A_{c}+A_{v}\right)$ in Fig. 1 and $1.09\left(A_{c}+A_{v}\right)$ in Fig. 2. It is seen that a relatively good agreement with the experimental points is found, thus indicating that the exponentially increasing absorption edge is due to the thermal

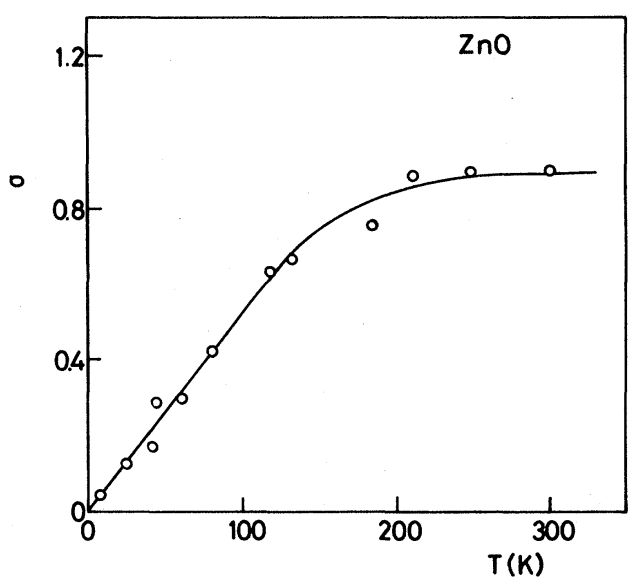

FIG. 3. Steepness parameter $\sigma$ in Urbach's rule for $\mathrm{ZnO}$ as a function of temperature. The experimental points are taken from Ref. 12. The solid curve is obtained by means of a fit with two different phonon modes (Ref. 12). 
fluctuations in the band-gap energy (exciton energy) of $\mathrm{ZnO}$.

A much better agreement is, however, obtained by applying the two-phonon parameter set giver. above. When two different interactions and vibration frequencies contribute, the band-gap shift of (35) can be written

$E_{g}(T)=E_{g}-3\left(A_{c 1}+A_{v 1}\right)\left\langle n_{1}\right\rangle-3\left(A_{c 2}+A_{v 2}\right)\left\langle n_{2}\right\rangle$.

From (33) and (34) it is found that

$$
\sigma=k T /\left(A_{c}+A_{v}\right)\left(\langle n\rangle+\frac{1}{2}\right) .
$$

Hence, (41) can be expressed in terms of $\sigma_{1}$ and $\sigma_{2}$ from the two-phonon parameter set as follows:

$$
\begin{aligned}
& E_{g}(T)=E_{0}^{\prime \prime}-3 k T\left(1 / \sigma_{1}+1 / \sigma_{2}\right), \\
& E_{0}^{\prime \prime}=E_{g 0}+\frac{3}{2}\left(A_{c 1}+A_{v 1}\right)+\frac{3}{2}\left(A_{c 2}+A_{v 2}\right) .
\end{aligned}
$$

With the parameters $\sigma_{01}=0.90, \sigma_{02}=2.20, \hbar \omega_{1}$ $=17 \mathrm{meV}$, and $\hbar \omega_{2}=72 \mathrm{meV}$ introduced into (33), the band-gap shift expected according to (43) is computed. The results are shown as the solid lines in Figs. 1 and 2.

Again the curves were shifted along the energy axis to give the best fit. This implies that $E_{0}^{\prime \prime}-E_{g 0}$ $=78.6 \mathrm{meV}$ in Fig. 1 and $E_{0}^{\prime \prime}-E_{g 0}=84.5 \mathrm{meV}$ in Fig. 2. It is seen that a better agreement with the experimental points is obtained in this case. It is best at higher temperatures for the band-gap shift due to the self energies alone. This could also be expected because of the simplified treatment of the contribution from the crystal expansion. The agreement obtained in Fig. 2 indicates, however, that the exponential absorption tail is due to the thermal fluctuations in the electron and hole selfenergy.

When there are different interactions and different vibration frequencies involved, as above, the simplest procedure is probably to assume that either one type of interaction (vibration mode) or another is present at a certain time in a certain space region. This will imply that (19) leads to the following generalization of Urbach's rule:

$$
\alpha=\sum_{i} \alpha_{0 i} \exp \left(-\frac{\sigma_{i}\left(E_{0 i}-h \nu\right)}{k T}\right),
$$

where $\sigma_{i}$ and $E_{0 i}$ are given by expressions analogous to (33) and (27). $\alpha_{0 i}$ is proportional to the dipole matrix element squared in (19) which is different for the different interactions involved. Similarly (43) may be generalized to

$$
E_{g}(T)=E_{00}-3 k T \sum_{i} \frac{1}{\sigma_{i}},
$$

where $E_{00}$ is given by an expression analogous to (44). Equation (45) was already applied in Ref. 12 to analyze the absorption edge of $\mathrm{ZnO}$ in terms of two types of phonons.

In a sum of exponential functions like (45) usually one of the terms will be much larger than the others, and the transition from one term to another will occur very abruptly. This property of a sum of exponentials makes comparison between the steepness parameters $\sigma_{i}$ and the band-gap shift difficult, since in a certain temperature range (and/or spectral range) only one exponential function, i.e., one $\sigma$ value, determines the absorption edge, while the sum of all the reciprocal $\sigma$ values determines the band-gap temperature shift.

Finally, it should be mentioned that the $\sigma_{0}$ values observed experimentally for different materials ${ }^{2}$ seem to fall into two groups. One group has $\sigma$ values close to 1 , and the other group has $\sigma$ values about 2-2.5. The first group consists mainly of I-VII compounds and relatively ionic crystals, while many II-VI compounds belong to the second group. It is tempting to assign optical-phonon interaction to one group and acoustical-phonon interaction to the other, as in the case of $\mathrm{ZnO}$ described above. ${ }^{12}$ This would mean that the electron interaction with optical phonons should give $\sigma$ values close to 2.5 , while acoustic-phonon interaction implies $\sigma$ values around 1 .

\section{CONCLUSION}

It has been shown that the thermal fluctuations in the phonon concentration lead to a fluctuating band-gap energy resulting in an exponentially increasing absorption edge near the band gap (Urbach's rule). The parameters in Urbach's rule were expressed in terms of parameters entering the band-gap shift with temperature, thus avoiding any specific assumptions about the nature of the electron-phonon interaction. Comparison with experimental results for $\mathrm{ZnO}$ shows good agreement at higher temperatures.

\section{APPENDIX A}

The most important interactions between electron and lattice are usually described in terms of the deformation potential interaction with acoustic phonons and the pure electrical interaction with optical phonons. The corresponding Hamiltonians (in mksa units) are given by

$$
\begin{aligned}
& \hat{H}_{\mathrm{LA}}=i C\left(\frac{\hbar}{2 M N}\right)^{1 / 2} \sum_{k, q} \frac{|q|}{\left(\omega_{q}\right)^{1 / 2}}\left(a_{q}-a_{-\alpha}^{\dagger}\right) c_{k+q}^{\dagger} c_{k}, \\
& \hat{H}_{\mathrm{LO}}=i e\left(\frac{\hbar \omega_{\mathrm{LO}}}{2 V \epsilon_{0} \epsilon^{*}}\right)^{1 / 2} \sum_{k, q} \frac{1}{|q|}\left(a_{q}-a_{q}^{\dagger}\right) c_{k+q}^{\dagger} c_{k},
\end{aligned}
$$

where $C$ is the deformation potential for the interaction with LA phonon, $M$ is the atomic mass, $N$ 
the number of unit cells, $q$ the phonon wave vector, and $\omega$ the phonon angular frequency. $a$ is the phonon-annihilation operator [LA in (A1) and LO in (A2)], while $c$ is the electron annihilation operator. $e$ is the electronic charge, $V$ the crystal volume, $\epsilon$ vacuum permittivity, $\epsilon^{*}=\left(1 / \epsilon_{0}-1 / \epsilon_{s}\right)^{-1}$, where $\epsilon_{0}$ and $\epsilon_{s}$ are the values of the dielectric constant at optic and low frequencies, respectively.

From (A1) and (A2) it is seen that the energy shift is zero in first-order perturbation theory. To second order the results at high temperatures for the sum of the electron and hole self-energies are given $b^{7}$

$$
\begin{aligned}
E_{\mathrm{LA}}= & -\frac{\hbar}{2 M v_{s}} \frac{\Omega}{(2 \pi)^{3}}\left(\frac{k T}{\hbar v_{s}} 8 \pi q_{\max }+2 \pi q_{\max }^{2}\right) \\
& \times\left(\frac{2 m_{e}}{\hbar^{2}} C_{e}^{2}+\frac{2 m_{h}}{\hbar^{2}} C_{h}^{2}\right), \\
E_{\mathrm{LO}}= & -\left(\alpha_{e}+\alpha_{h}\right) \hbar \omega_{\mathrm{LO}}(n+1) \\
\simeq & -\left(\alpha_{e}+\alpha_{h}\right)\left(k T+\hbar \omega_{\mathrm{LO}}\right),
\end{aligned}
$$

where $v_{s}$ is the velocity of the LA phonons, $\Omega$ is the unit cell volume, $k T$ is the thermal energy, $q_{\max }^{3}=3(2 \pi)^{3} /(4 \pi \Omega)$ is the maximum $q$ value, and $m_{e}$ and $m_{h}$ are the electron and hole masses; while $C_{e}$ and $C_{h}$ are deformation potential constants for conduction and valence band, respectively;

$$
\alpha_{e, h}=\left(e^{2} / 4 \pi \epsilon_{0} \epsilon^{*} \hbar\right)\left(m_{e, h} / 2 \hbar \omega_{\mathrm{LO}}\right)^{1 / 2}
$$

is the polaron coupling constant for the electron $(e)$ and hole $(h)$. Equations (A3) and (A4) are the contributions to the band-gap shift with temperature from deformation potential (A3) and polaron coupling (A4).

For ZnO the relevant parameters are $M=1.36$ $\times 10^{-25} \mathrm{~kg}, v_{s}=6.1 \times 10^{3} \mathrm{~m} / \mathrm{sec}^{15} \Omega=4.73 \times 10^{-29}$ $\mathrm{m}^{3},{ }^{16} m_{e}=0.28 m_{0}{ }^{17} m_{h}=0.74 m_{0}$ (polaron masses), ${ }^{18}$ and $C=4.0 \mathrm{eV}$ [from Eq. (39)]. From (A3) we then find, by replacing $\left(2 m_{e} C_{e}^{2}+2 m_{h} C_{h}^{2}\right) / \hbar^{2}$ by $2\left(m_{e}+m_{n}\right) C^{2} / \hbar^{2}$,

$$
\left(\frac{d E g}{d T}\right)_{\mathrm{LA}}=-0.030 \mathrm{meV} / K \text {. }
$$

With $\epsilon^{*}=8.1,^{3} m_{e}=0.24 m_{0},^{17} m_{h}=0.59 m_{0}$ (bare masses, ${ }^{18}$ and $\hbar \omega_{\mathrm{LO}}=72 \mathrm{meV},{ }^{3}$ one obtains $\alpha_{e}$ $=0.83$ and $\alpha_{h}=1.30$. From (A4) one then finds

$$
\left(\frac{d E_{g}}{d T}\right)_{\text {LO }}=-0.18 \mathrm{meV} / \mathrm{K} \text {. }
$$

The values obtained in (A5) and (A6) should be compared with the high-temperature slope of the experimental band-gap shift (corrected for crystal expansion) in Fig. 2. This slope is found to be $d E_{g} / d T=-0.27 \mathrm{meV} / \mathrm{K}$. It is thus seen that neither the deformation potential interaction nor the polaron contribution nor the sum of these are sufficient to explain the value of the band-gap shift observed experimentally.

A best fit to the experimental points in Fig. 2 of an expression of the form $E_{g}(T)=E_{g 0}-A$ / $\left(e^{\hbar \omega / k T}-1\right)$, with ${ }^{11} E_{g 0}=3.4210 \mathrm{eV}$ yields $A=82.6$ $\mathrm{meV}$ and $\hbar \omega=23.7 \mathrm{meV}$, indicating that both acoustic and optical phonons contribute to the band-gap shift, since $23.7 \mathrm{meV}$ is between opticaland acoustic-phonon energies. With two types of phonons an expression of the form

$E_{g}(T)=E_{g 0}-A_{1} /\left(e^{\hbar \omega_{1} / k T}-1\right)-A_{2} /\left(e^{\hbar \omega_{2} / k T}-1\right)$

can be used for fitting. A best fit to the experimental points in Fig. 2 yields $A_{1}=44.2$ and $A_{2}$ $=104.5 \mathrm{meV}$, when $E_{g 0}=3.4210 \mathrm{eV}, \hbar \omega_{1}=17 \mathrm{meV}$, and $\hbar \omega_{2}=72 \mathrm{meV}$. This means that the experimental results seem to indicate that the contribution from the acoustic phonons at high temperatures is

$$
\left(\frac{d E_{g}}{d T}\right)_{A}=-\frac{k A_{1}}{\hbar \omega_{1}}=-0.22 \mathrm{meV} / \mathrm{K}
$$

and from the optical phonons

$$
\left(\frac{d E_{g}}{d T}\right)_{0}=-\frac{k A_{2}}{\hbar \omega_{2}}=-0.12 \mathrm{meV} / \mathrm{K} \text {. }
$$

Similar estimates for the two contributions can be obtained from the values $\sigma_{01}=0.90$ and $\sigma_{02}=2.20$ quoted in Sec. IV. Applying (43) it is then seen that

$$
\left(\frac{d E_{g}}{d T}\right)_{A}=-\frac{3 k}{\sigma_{01}}=-0.28 \mathrm{meV} / \mathrm{K}
$$

and

$$
\left(\frac{d E_{g}}{d T}\right)_{0}=-\frac{3 k}{\sigma_{02}}=-0.12 \mathrm{meV} / \mathrm{K} .
$$

Equations (A10) and (A11) are in good agreement with the values (A8) and (A9) obtained from the two-phonon fit to the band-gap shift. Hence, it can be concluded that the part of the band-gap shift due to optical phonons seems relatively well described by the polaron contribution (A6) although this value is somewhat too large, probably because of the neglection of exciton effects. The shift due to the acoustical phonons can not be explained in terms of the deformation potential interaction with LA phonons alone, since the value (A5) is a factor of 10 too sinall. The piezoelectric self-energy is still a magnitude smaller. ${ }^{19}$ Then the interaction with the TA phonons remains. Such an interaction may be important since shear strain tend to split the valence bands and thus introduce a temperature-dependent self-energy for the holes.

\section{APPENDIX B}

In the model outlined in Sec. III there exists a relationship between the first-order and second- 
order shift of the local band gap in (26). Hence, if the local band gap is much smaller than the value given in (31), it is dominated by the quadratic term in (26). In order to estimate the value in (31), $A_{c}, A_{v}$, and $\Delta_{0}$ must be known. From the results in Sec. IV one obtains $A_{c}+A_{v}=33.3 \mathrm{meV}$ from the one-phonon parameter set which will be applied in the following. $A_{c}$ enters Eq. (28) and may be estimated from this, since it determines the linewidth of the absorption line. In analogy with the derivation in Sec. III one obtains for the absorption coefficient, if the quadratic term in (26) is neglected and $\vec{\Delta}_{0}$ is treated as a scalar $\left(\left|\vec{\Delta}_{\mathrm{o}}\right|\right)$,

$\alpha=\alpha_{0} \exp \left[-\left(E_{0}-h \nu\right)^{2} \tanh \left(\frac{\hbar \omega}{2 k T}\right) / F^{2} \alpha^{2} \Delta_{0}^{2}\right]$,

where $\alpha_{0}$ has the same value as in (32). Hence, the $\alpha_{0}$ parameter of Urbach's rule can be interpreted as the maximum absorption coefficient at the center of the absorption line representing the band gap (i.e., the ground-state exciton line).

The half width of this line is given by

$$
\begin{aligned}
\Delta E & =2(\ln 2 \operatorname{coth} \hbar \omega / 2 k T)^{1 / 2} F \alpha \Delta_{0} \\
& \simeq 2 A_{c}[(2 k T / \hbar \omega) \ln 2]^{1 / 2}\left(M \omega \Delta_{0}^{2} / \hbar\right)
\end{aligned}
$$

for $k T \gg \hbar \omega$.

From (21) and (25) an estimate of $\Delta_{0}$ can be obtained. Consider, for example, an emission process with an electron in the upper level, and let us assume that the lattice is relaxed so that no phonons are present (i.e., $\Delta_{n c} \simeq \Delta_{0}$ ). When the emission process occurs $\Delta_{n c}$ is unchanged as discussed in Sec. III, and (25) shows that the lattice, after the transition has occurred, is excited with $n_{v}$ phonons present where $n_{v} \hbar \omega \simeq \frac{1}{2} M \omega^{2} \Delta_{0}^{2}$. Hence, during the emission process there will in average be emitted $n_{v}$ phonons of energy $\hbar \omega$. It is well known from the phonon-assisted edge emission spectra that the average number of phonons emitted is of about $0.5-1$ for most II-VI compounds. In the estimate of $\Delta_{0}$ we shall therefore assume that $M \omega \Delta_{0}^{2} / \hbar \simeq 1$. A value for $A_{c}$ can then be obtained from the width of the exciton line. At $T$ $=300 \mathrm{~K}$ the halfwidth of the $C$-exciton line is 50 meV. ${ }^{11}$ From (A2) one then finds $A_{c}=23 \mathrm{meV}$. From (31) it is now possible to estimate in which spectral region the quadratic term in (26) is dominating, resulting in an absorption tail which is purely exponential,

$$
\begin{aligned}
h \nu & \ll E_{g 0}+50 \mathrm{meV}-74.3 \mathrm{meV} \\
& =E_{g 0}-24 \mathrm{meV} \simeq 3.39 \mathrm{eV} .
\end{aligned}
$$

Here, the ground-state energy of the $C$ exciton [3.4210 eV (Ref. 11)] has been used for $E_{g 0}$. In Ref. 12 the spectral region near $3.40 \mathrm{eV}$ is only reached for the very lowest temperatures; and for these temperatures there are deviations from the exponential behavior.

With the (rough) estimate of $\Delta_{0}$ given above it is also possible to give an estimate of the $E_{0}$ parameter in Urbach's rule as given by (27)

$$
\begin{aligned}
E_{0} & =E_{g 0}+\frac{3}{2}\left(A_{c}+A_{v}\right)-A_{c}(M \omega / 2 \hbar) \Delta_{0}^{2} \\
& =E_{g 0}+38.5 \mathrm{meV}=3.46 \mathrm{eV} .
\end{aligned}
$$

From experiments ${ }^{12}$ a value of $3.51 \mathrm{eV}$ was found. It is, however, difficult to determine $E_{0}$ experimentally (from extrapolations of the isoabsorption curves). If $E_{0}=3.46 \mathrm{eV}$ is used instead of $3.51 \mathrm{eV}$, the value of $\alpha_{0}=4.5 \times 10^{6} \mathrm{~cm}^{-1}$ quoted in Ref. 12 will be reduced to $\alpha_{0}=4.7 \times 10^{5} \mathrm{~cm}^{-1}$. This value corresponds better to the experimental value of ${ }^{20}$ $5 \times 10^{5} \mathrm{~cm}^{-1}$ for the maximum absorption coefficient at the $C$-exciton resonance at low temperatures.

Finally, it is seen from (26) that it is possible to estimate a value for a deformation potential constant defined as

$$
\begin{aligned}
|C|=\left|\frac{d E_{g}}{d(\Delta / a)}\right| & =F a \Delta_{0} \\
& =A_{c} a \frac{M \omega \Delta_{0}}{\hbar} \simeq A_{c} \frac{a}{\Delta_{0}},
\end{aligned}
$$

where $a$ is the average lattice constant. Here, it has been used that $M \omega \Delta_{0}^{2} / \hbar \simeq 1$, from which $\Delta_{0}$ $=0.041 \AA$ is obtained (with $\hbar \omega=30 \mathrm{meV}$ and $M=1.36 \times 10^{-25} \mathrm{~kg}$ ). The average lattice constant is found as

$$
d=(\Omega)^{1 / 3}=\left(4.73 \times 10^{-23} \mathrm{~cm}^{3}\right)^{1 / 3}=3.62 \AA .
$$

With these values introduced into (B3) we find

$$
C=2.0 \mathrm{eV} \text {, }
$$

which is the right order of magnitude and only a factor of 2 less than the isotropic value found in (39). From (26) it is seen that a second order deformation potential constant also may be defined as

$$
\begin{aligned}
\left|C^{\prime \prime}\right|=\left|\frac{d^{2} E_{g}}{d(\Delta / a)^{2}}\right| & =2 G a^{2} \\
& =\frac{M \omega \Delta_{0}^{2}}{\hbar}\left(A_{c}+A_{v}\right)\left(\frac{a}{\Delta_{0}}\right)^{2} \\
& =257 \mathrm{eV} .
\end{aligned}
$$


${ }^{1}$ F, Urbach, Phys. Rev. 92, 1324 (1953).

${ }^{2}$ M. V. Kurik, Phys. Status Solidi. A 8, 9 (1971).

${ }^{3}$ J. D. Dow and D. Redfield, Phys. Rev. B 5, 594 (1972).

${ }^{4} \mathrm{H}$. Sumi and Y. Toyozawa, J. Phys. Soc. Jpn. 31,342 (1971).

${ }^{5}$ R. C. Tolman, The Principles of Statistical Mechanics (Oxford, London, 1938).

${ }^{6}$ R. J. Collins and D. A. Kleinman, J. Phys. Chem. Solids 11,190 (1959).

${ }^{7}$ H. Y. Fan, Phys. Rev. 82,900 (1951).

${ }^{8}$ Y. F. Tsay, S. S. Mitra, and J. F. Vetelino, J. Phys. Chem. Solids 34, 2167 (1973).

${ }^{9}$ J. J. Markham, Rev. Mod. Phys. 31, 956 (1959).

${ }^{10}$ D. Pines, in Polarons and Excitons, edited by G. D. Whitfield (Oliver and Boyd, Edinburgh, 1963), p. 33.

${ }^{11}$ G. H. Jensen, Phys. Status Solidi B 64, K51 (1974).

${ }^{12}$ G. H. Jensen and T. Skettrup, Phys. Status Solidi B $\underline{60}$,
169 (1973)

${ }^{13}$ B. Yates, R. F. Cooper, and M. M. Kreitman, Phys. Rev. B 4, 1314 (1971).

${ }^{14}$ R. L. Knell and D. W. Langer, Phys. Lett. 21, 370 (1966).

${ }^{15}$ T. B. Bateman, J. Appl. Phys. 33, 3309 (1962).

${ }^{16}$ G. Heiland, E. Mollwo, and F. Stöckmann, in Solid State Physics, edited by F. Seitz and D. Turnbull (Academic, New York, 1959), Vol. 8, p. 193.

${ }^{17}$ K. J. Button, D. R. Cohn, M. V. Ortenberg, B. Lax, E. Mollwo, and R. Helbig, Phys. Rev. Lett. 28, 1637 (1972).

${ }^{18} \mathrm{~K}$. Hümmer, Phys. Status Solidi B 56, 249 (1973).

${ }^{19}$ C. E. Bleil, J. Phys. Chem. Solids $\overline{27}, 1631$ (1966).

${ }^{20}$ W. Y. Liang and A. D. Yoffe, Phys. Rev. Lett. 20, 59 (1968). 